\title{
Direct demonstration of spatial water distribution in the sponge Suberites domuncula by in vivo NMR imaging
}

\author{
Gerhard Bringmann ${ }^{1, *}$, Kristina Wolf ${ }^{1}$, Titus Lanz ${ }^{2}$, Axel Haase ${ }^{2}$, Jan Hiort ${ }^{3}$, Peter Proksch ${ }^{3}$, \\ Werner E. G. Müller ${ }^{4}$ \\ ${ }^{1}$ Institut für Organische Chemie and ${ }^{2}$ Lehrstuhl für Experimentelle Physik V, Universität Würzburg, Am Hubland, 97074 Würzburg, \\ Germany \\ ${ }^{3}$ Julius-von-Sachs-Institut für Biowissenschaften, Universität Würzburg, Julius-von-Sachs-Platz 2, 97082 Würzburg, Germany \\ ${ }^{4}$ Institut für Physiologische Chemie, Abteilung Angewandte Molekularbiologie, Johannes-Gutenberg-Universität Mainz, \\ Duesbergweg 6, 55099 Mainz, Germany
}

\begin{abstract}
Until now, direct, non-invasive in vivo studies on water and metabolite distribution in living sponges have not been possible. Here we apply for the first time the noninvasive technique of nuclear magnetic resonance (NMR) imaging to determine the spatial distribution of water in the marine sponge Suberites domuncula. After transfer of the sponge into deuterated water $\left(\mathrm{D}_{2} \mathrm{O}\right)$ for a short incubation period of $18 \mathrm{~min}$, no significant water exchange was observed, neither in $S$. domuncula nor in the hermit crab living in symbiosis with it, suggesting $\mathrm{D}_{2} \mathrm{O}$ to be an ideal contrast enhancing agent for NMR imaging of sponges. Thus, NMR imaging provides a promising technique for the detection (and possibly quantification) of the distribution and transport of water both by diffusion and active transport in a living sponge.
\end{abstract}

KEY WORDS: NMR imaging - Sponges - Suberites domuncula . Water distribution

Porifera (sponges) represent the most simple metazoan phylum. They possess a remarkably developed aquiferous system of water transport channels and choanocyte chambers; this system is essential for food uptake, gas exchange, and reproduction (reviewed in: Simpson 1984). As filter feeders, some sponge species filter $24000 \mathrm{l} \mathrm{kg}^{-1} \mathrm{~d}^{-1}$ (Reiswig 1974). First approaches to visualize the 3-dimensional reconstruction of the channel system have been performed using the corrosion cast technique (Bavestrello et al. 1998). During the application of this technique, however, the sponge

•E-mail: bringman@chemie.uni-wuerzburg.de specimens are destroyed so that no repeated or even continuous time-dependent measurements are possible. Moreover, quantitative information that is required for a detailed biophysical analysis of the water flow is lacking. Furthermore, the morphogenesis of the channel system, which appears to be the major process during growth and development, is only poorly understood (reviewed in: Simpson 1984).

Sponges have recently gained wider interest for 3 major reasons. Firstly, they are rich sources of a broad variety of bioactive compounds (reviewed in: Sarma et al. 1993) that are used e.g. as a chemical defence against predators such as carnivorous fish (reviewed in: Proksch 1994). Secondly, the elucidation of the genome composition and organization of sponges as 'living fossils' allows insight into the early evolution of Metazoa prior to the Cambrian Explosion (reviewed in: Müller 1998). Thirdly, cultivation of marine sponges in bioreactors (Osinga et al. 1998) and in vitro (Müller et al. 1999) has recently become possible for the first time. One major limitation in the process of optimizing the culture conditions, especially with respect to the growth of the samples in vitro, is the lack of a reliable and quantitative technique to analyze the water flow in living specimens.

In the present study the non-invasive technique of in vivo nuclear magnetic resonance (NMR) imaging (reviewed in: Callaghan 1991) is introduced to determine for the first time the distribution of water in a living sponge, using, as an example, the marine sponge Suberites domuncula. This species is suitable for the application of the NMR imaging technique since it filters only $200 \mathrm{l} \mathrm{kg}^{-1} \mathrm{~d}^{-1}$ of water and consumes 

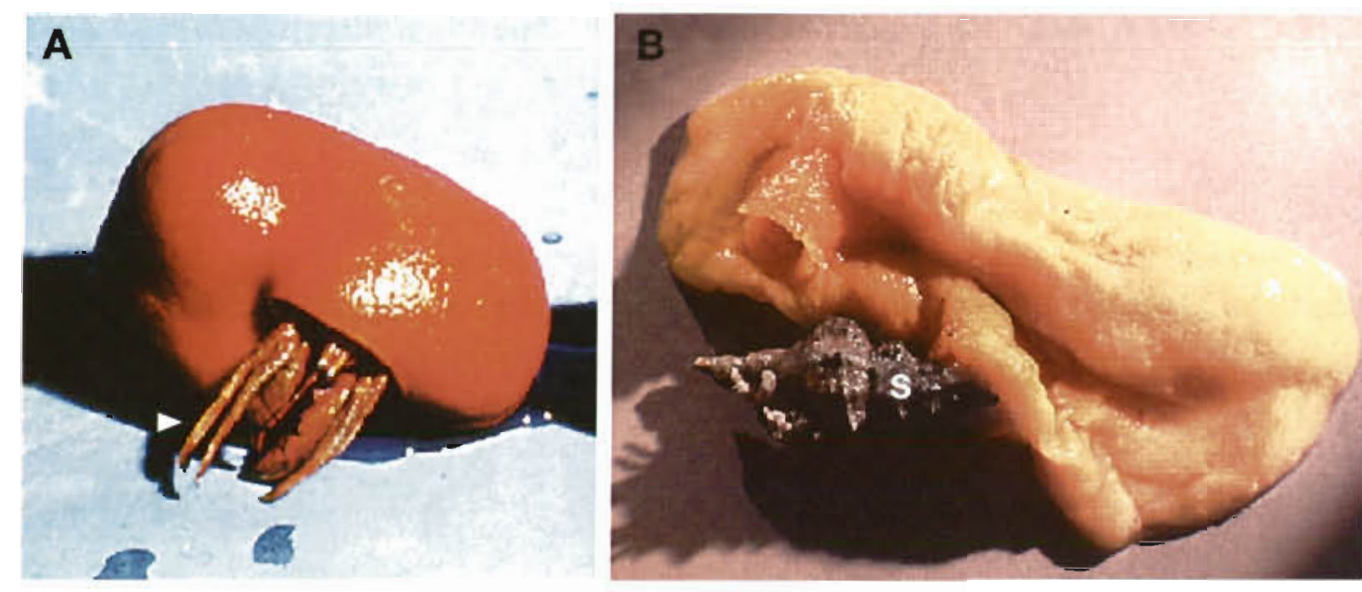

Fig. 1. Marine sponge Suberites domuncula. (A) A specimen covering a mollusc shell with a hermit crab; the pereopods, chelipeds and 1 antenna of the crab are visible (arrowhead). In (B) the sponge tissue that surrounds the shell (S) has been removed ca $240 \mathrm{mg} \mathrm{kg} \mathrm{kg}^{-1} \mathrm{O}_{2} \mathrm{~d}^{-1}$ at a temperature of $10^{\circ} \mathrm{C}$ (Arndt 1930). In addition, $S$. domuncula is a particularly interesting object since it lives in symbiosis with a hermit crab, and, in consequence, images are taken simultaneously from 2 living animal species, with the shell of a (dead) third organism (a snail) as a 'reference'.

Materials and methods. Animals: Suberites domuncula (Porifera: Demospongiae: Tetractinomorpha: Hadromerida: Suberitidae: Suberites) were collected in September 1998 from a depth of $22 \mathrm{~m}$ near Rovinj (Croatia). All specimens are known to live in association with the hermit crab Pagurites oculatus (Decapoda: Paguridea) (Fig. 1A), which resides in shells of the mollusc Trunculariopsis trunculus (Gastropoda: Muricidae) (Fig. 1B). The specimens of $S$. domuncula may reach a size of $10 \mathrm{~cm}$ (in diameter) with an average of $4.5 \mathrm{~cm}$.

NMR imaging: The NMR imaging experiments were performed using a Bruker BIOSPEC 70/20 NMR spectrometer with a magnetic field strength of $7 \mathrm{~T}$ and a horizontal bore of $20 \mathrm{~cm}$ (Bruker Medical $\mathrm{GmbH}$, Rheinstetten, Germany). The actively shielded gradient coils were capable of achieving gradients up to 200 Gauss $\mathrm{cm}^{-1}$, with rise and fall times of $240 \mu \mathrm{s}$. For excitation and detection of the NMR signal at the ${ }^{1} \mathrm{H}$ resonance frequency of $300.3 \mathrm{MHz}$, a home-built birdcage resonator (Hayes 1985) was used. The resonator is a high pass design with 8 rungs, a diameter of $37 \mathrm{~mm}$, and a length of $50 \mathrm{~mm}$. In order to improve the signalto-noise ratio (SNR) by a factor of 1.4 , the birdcage coil was quadrature driven (Chen et al, 1983).

For imaging, a 3-dimensional FLASH NMR imaging sequence (Haase 1986) with an excitation pulse of rectangular time pulse profile and a length of $200 \mu$ s was applied. A $128 \times 128 \times 8$ data matrix was used for a field of view of $35 \times 35 \times 25 \mathrm{~mm}$, resulting in an inplane resolution of $35 \mathrm{~mm} / 128=0.27 \mathrm{~mm}$ and in a slice thickness of $25 \mathrm{~mm} / 8=3.1 \mathrm{~mm}$ for each of the 8 individual slices. The echo time was $1.4 \mathrm{~ms}$, and the repe- tition time was $2.5 \mathrm{~ms}$. The number of averages was set to 16 (Fig. 3A) and 4 (Fig. 3B), resulting a total measurement time of 49.5 and $18.4 \mathrm{~s}$, respectively.

The specimen was placed into a glass tube $(28 \mathrm{~mm}$ outer diameter) filled either with seawater (Sigma) or with artificial seawater prepared from deuterated water $\left(\mathrm{D}_{2} \mathrm{O}\right)$. The incubation time for deuterated water was $18 \mathrm{~min}$. The tube was sealed and placed into the magnet horizontally. The experiments were performed at $20^{\circ} \mathrm{C}$.

Results. Distribution of water within the organism: First images were taken from a specimen kept in seawater. NMR slices were selected through the lower (Panel a), middle (Panel b), and upper (Panel c) parts of the animal (Fig. 2). Slice a contains the tip of the helical snail shell within the sponge tissue. The centers of both the sponge and the snail shell are located in Slice b, and Slice c covers the area towards the oscule.

The colorbar given in Fig. 3Aa correlates the artificial colors of the images with the NMR signal intensities of the detectable aqueous protons. This scale,

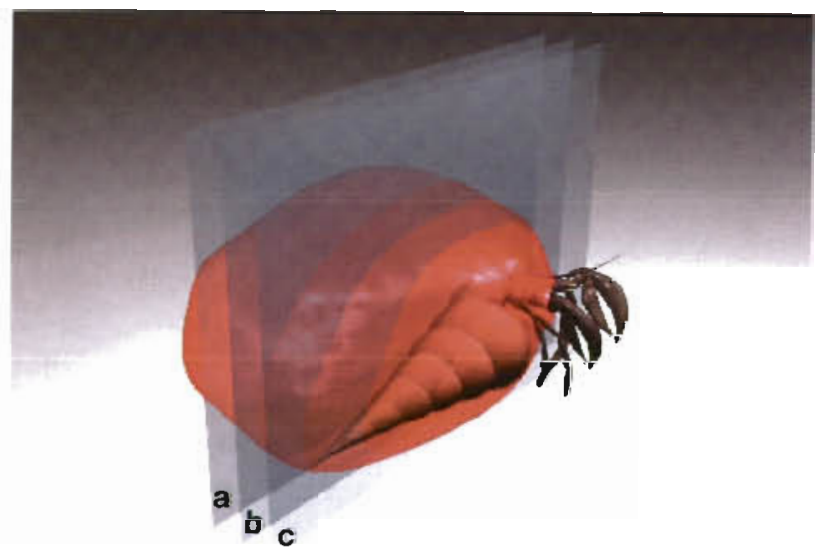

Fig. 2. Sketch showing the selection of the 3 NMR Slices $a, b$, and $\mathrm{c}$ through Suberites domuncula 
reaching from lowest (black) to highest (yellow) intensities, reflects the (relative) intensity distribution within a distinct NMR experiment and thus applies to all of the 3 images a to $\mathrm{C}$ shown in Fig. 3A. Due to the fast imaging method used, the images do not reflect the pure spin density, and thus not only the pure water content, but are weighted by the NMR relaxation rate, $T_{1}$. Thus, the high intensities stand for both high water content and short $T_{1}$. The SNR reaches 100 in Fig. 3Ab. Most of the sponge tissue regions are in green indicating an intermediate proton NMR signal intensity (about 60\%) relative to the surrounding water NMR signal. The ratio of the average signal intensities between seawater (high signal) and tissue (low signal) is 1.6. In one area, however, Fig. 3Ab displays a red color, which is indicative of a proton signal virtually identical to that in the surrounding medium. This region represents the area of the oscule The NMR contrast between the sponge tissue and its aqueous environment becomes even more obvious in Fig. 3Ac.

Distribution of water after hydrogen-deuterium exchange: After the above measurements, the specimen was transferred from water into $\mathrm{D}_{2} \mathrm{O}$. In proton NMR imaging, $\mathrm{D}_{2} \mathrm{O}$ does not give a NMR signal (due to its different resonance frequency) and is thus expected to appear as black in the image. Residual $\mathrm{H}_{2} \mathrm{O}$ held back inside the sponge and the snail shell during the transfer, however, became mixed with the surrounding $\mathrm{D}_{2} \mathrm{O}$ and gave rise to the blue color. The colorbar given in Fig. 3Ba holds only for images shown in Fig. 3B. The intensities in the 2 series of experiments do not follow the same absolute scale and are thus not directly comparable with one another. Direct comparability would require the addition of an external reference. The SNR was 50 for the image shown in Fig. 3Bb, half of the SNR measured for the image in Fig. $3 \mathrm{Ab}$, whereas the ratio of the signal intensities between the tissue (now high
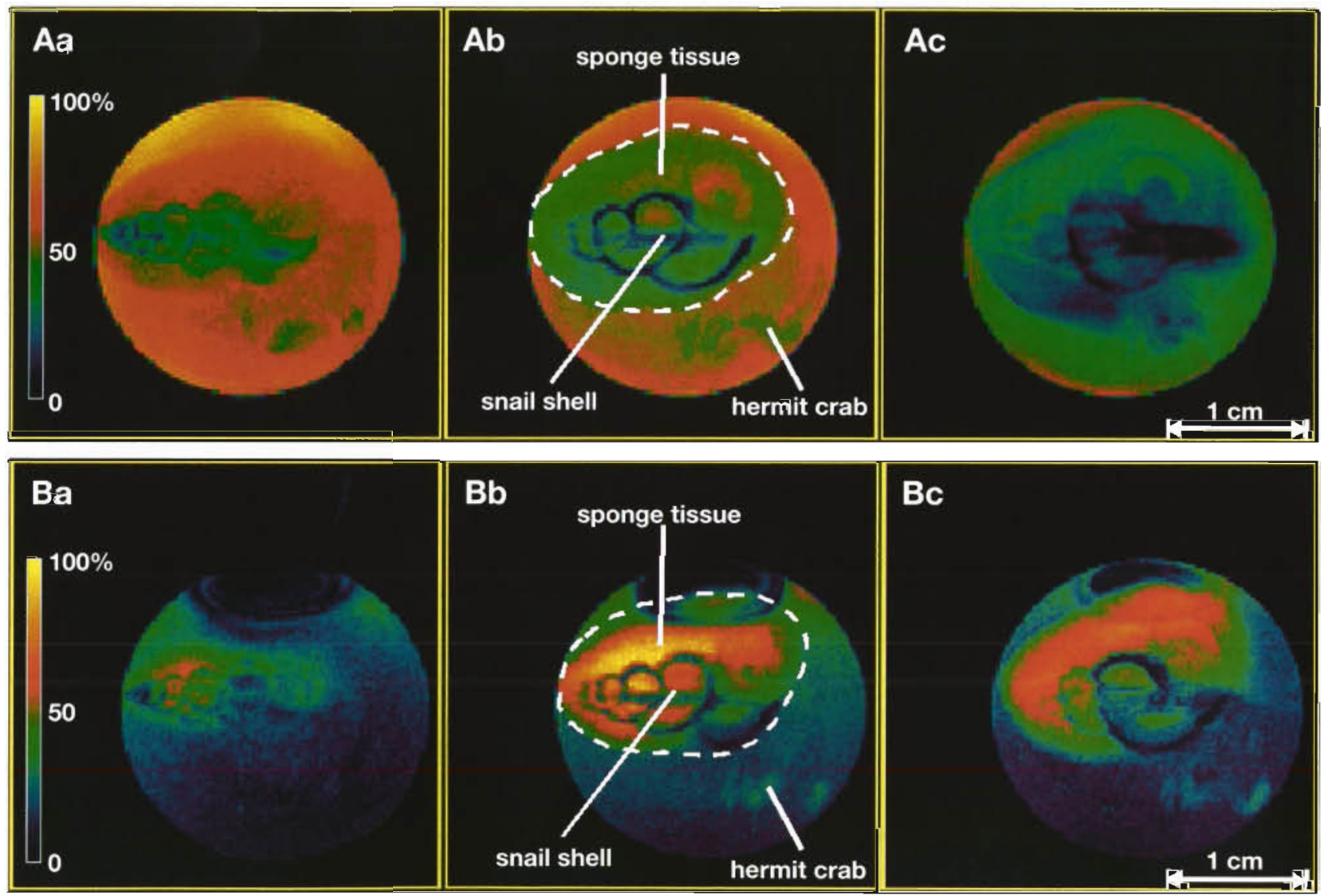

Fig. 3. NMR imaging of Suberites domuncula $(A)$ in seawater and $(B)$ in seawater composed of deuterated water $\left(D_{2} O\right)$. The images were taken from a specimen placed in a round glass tube using the 3-dimensional FLASH method. From the 8 NMR slices obtained, the 3 most informative ones are shown (slice thickness $3.1 \mathrm{~mm}$ ): $\mathbf{a}$, the slice preceding the central slice; $\mathbf{b}$, the image from the middle of the animal; and $\mathrm{c}$, the slice following the middle slice. In (Ab) and (Bb) the outer surface of the sponge is indicated by a dashed line; in addition, the locations of the shell, the legs of the crab, and of the sponge tissue are marked. The color bars (ranging from yellow to black) mark the NMR signal indicative of the relative $T_{1}$-weighted water content; note that the contrast of the images in (B) has been increased by about 1.5 -fold. Field of view: $35 \times 35 \mathrm{~mm}$; resolution: $0.27 \mathrm{~mm}$ 
signal) and the surrounding medium (now low signal) increased from 1.6 (Fig. 3Ab) to 2.5 (Fig. 3Bb). After an incubation period of $2 \mathrm{~min}$, as also required for the necessary manipulations, the water signal in the opening through which the crab in the shell reaches the surrounding medium with its legs is reduced to almost zero (Fig. 3Bb). Unexpected is the finding that the relative $\mathrm{H}_{2} \mathrm{O}$ signal intensity in the sponge tissue remains at an elevated level, even higher than the one measured in the crab (Fig. 3Bb). Only in the outer layer (ca $2 \mathrm{~mm}$ ) of the sponge does the color of the image turn green, indicating a reduction of the water signal intensity. It is interesting to note that the hermit crab in the shell shows the same relative water concentration as the sponge tissue. As in Fig. $3 \mathrm{AC}_{\text {, }}$ the slice shown in Fig. 3Bc exhibits the clearest contrast between sponge and environment.

Discussion. It was established that Porifera are to be grouped together with the other metazoan phyla into one monophyletic kingdom (Müller 1995). In consequence, it is also reasonable to assume that the outer surface of the sponges, the pinacoderm, maintains a higher degree of homeostasis, also with respect to the internal water content, with the surrounding medium than previously suggested (Loomis 1988). The data shown here demonstrate that the NMR signal intensity of water in the sponge remains at the same level as in the hermit crab after an incubation with $\mathrm{D}_{2} \mathrm{O}$ for a period of $18 \mathrm{~min}$.

These findings suggest $\mathrm{D}_{2} \mathrm{O}$ as an ideal contrast enhancing agent for NMR imáging of sponges. Based on these experiments, further NMR imaging studies offer the possibility of determining the water content in sponges quantitatively. Taking advantage of the main strength of NMR imaging, the non-invasivity, functional parameters, such as water flow and diffusion, might be recorded for a living organism. Furthermore, the use of NMR contrast agents, e.g. paramagnetic complex reagents used in medicine to enhance $T_{t}$ contrast, and tracer substances should offer additional possibilities both for anatomic and metabolic studies in vivo.

Acknowledgements. This work was funded by BMBF (Bundesrepublik Deutschland) and ASTA Medica AG (Förder-

Editorial responsibility: Otto Kinne (Editor),

Oldendorf/Luhe, Germany kennzeichen 03F0239A4), the Fonds der Chemischen Industrie, and the Deutsche Forschungsgemeinschaft (Graduiertenkolleg 'NMR in vivo und in vitro für die biologische und medizinische Grundlagenforschung'). K.W. thanks the Studienstiftung des Deutschen Volkes for a generous fellowship. The authors wish to thank Dr Markus Heubes, Heike Friedrich, and Petra Rösch for fruitful discussions.

\section{LITERATURE CITED}

Arndt W (1930) Schwämme (Porifera, Spongien). Tabulae Biol VI(Suppl II):41-120

Bavestrello G, Cerrano C, Corriero G, Sara M (1998) Threedimensional architecture of the canal system of some hadromerids (Porifera, Demospongiae). In: Watanabe Y, Fusetani $N$ (eds) Sponge science-multidisciplinary perspectives. Springer-Verlag, Tokyo, p 235-247

Callaghan PT (1991) Principles of nuclear magnetic resonance microscopy. Clarendon Press, Oxford

Chen CN, Hoult DI, Sank VJ (1983) Quadrature detection coils - a further $\sqrt{ } 2$ improvement in sensitivity. J Magn Reson 54:324-327

Haase A, Frahm J, Matthaei D, Hänicke W, Merboldt KD (1986) FLASH imaging. Rapid NMR imaging using low flip-angle pulses. J Magn Reson 67:258-266

Hayes CE, Edelstein WA, Schenck JF, Mueller OM, Eash M (1985) An efficient, highly homogeneous radiofrequency coil for whole-body NMR imaging at 1.5 T. J Magn Reson 63:622-628

Loomis WF (1988) Four billion years: an assay on the evolution of genes and organisms. Sinnauer Assoc Publishers Sunderland, MA

Müller WEG (1995) Molecular phylogeny of Metazoa (animals): monophyletic origin. Naturwissenschaften 82 321-329

Müller WEG (1998) Origin of Metazoa: sponges as living fossils. Naturwissenschaften 85:11-25

Müller WEG, Wiens M, Batel R. Steffen R, Schröder HC, Borojevic R, Custodio MR (1999) Establishment of a primary cell culture from a sponge: primmorphs from Suberites domuncula. Mar Ecol Prog Ser 178:205-219

Osinga R, Tramper J, Wijffels RH (1998) Cultivation of marine sponges for metabolite production: application for biotechnology? Trends Biotechnol 16:130-134

Proksch P (1994) Defensive roles for secondary metabolites from marine sponges and sponge-feeding nudibranchs. Toxicon 43:639

Reiswig HM (1974) Water transport, respiration, and energetics of three topical sponges, J Exp Mar Biol Ecol 14:231-249

Sarma AS, Daum T, Müller WEG (1993) Secondary metabolites from marine sponges. Ullstein-Mosby Verlag. Berlin, p $1-168$

Simpson TL (1984) The cell biology of sponges. SpringerVerlag, New York

Submitted: August 2, 1999; Accepted: October 13, 1999

Proofs received from author(s): October 27, 1999 